\section{$\underset{\substack{\text { hommes } \\ \text { \& migrations }}}{ }$}

\section{Hommes \& migrations}

Revue française de référence sur les dynamiques

migratoires

1303 | 2013

Diasporas marocaines

\title{
Deux décennies de transnationalisme associatif : continuités et changement
}

\section{Thomas Lacroix}

\section{(apenEdition \\ Journals}

\section{Édition électronique}

URL : http://journals.openedition.org/hommesmigrations/2565

DOI : 10.4000/hommesmigrations.2565

ISSN : 2262-3353

\section{Éditeur}

Musée national de l'histoire de l'immigration

\section{Édition imprimée}

Date de publication : 1 juillet 2013

Pagination : 101-110

ISBN : 978-2-919040-23-0

ISSN : $1142-852 X$

\section{Référence électronique}

Thomas Lacroix, « Deux décennies de transnationalisme associatif : continuités et changement », Hommes \& migrations [En ligne], 1303 | 2013, mis en ligne le 31 décembre 2015, consulté le 20 avril 2019. URL : http://journals.openedition.org/hommesmigrations/2565; DOI : 10.4000/ hommesmigrations.2565 


\section{DEUX DÉCENNIES \\ DE TRANSNATIONALISME ASSOCIATIF CONTINUITÉS ET CHANGEMENT}

par THOMAS LACROIX, chargé de recherche au CNRS, Migrinter, université de Poitiers, chercheur associé, International Migration Institute, université d'Oxford

L'engagement associatif en faveur du pays d'origine est l'un des principaux chantiers des associations d'immigrés marocains en France. Autrefois centrés sur l'amélioration des conditions de vie dans le pays d'accueil, les projets associatifs sont désormais consacrés en majorité au développement social des régions dont les migrants sont originaires. Le dynamisme de ses différentes composantes sur le territoire français permet à ce réseau associatif de mener à bien de véritables projets transnationaux.

\section{Histoire de l'associationnisme des immigrés marocains en France}

Il y a dix ans un article paru dans le numéro spécial sur les migrations marocaines d'Hommes \& Migrations $^{1}$ relevait la structuration pyramidale des réseaux associatifs marocains et franco-marocains engagés dans des initiatives de développement Nord-Sud. À la base de cette pyramide se trouvait un ensemble plus ou moins formel de petites associations et collectifs, maîtres d'œuvre de petits projets d'infrastructures pour leur village ou région d'origine. Au-dessus, opéraient une poignée d'associations fédératives faisant le lien entre ces porteurs de projets et les bailleurs de fonds publics ou privés. Ces organisations, telles que Migrations et développement à Marseille ou Immigrations, démocratie, développement à Paris, sont animées par d'anciens syndicalistes et militants réfugiés de la gauche marocaine qui ont reconverti leur savoirfaire de mobilisation collective dans le domaine du développement.

Il s'agit aujourd'hui ${ }^{2}$ d'examiner le devenir de ce réseau, d'analyser les impacts de ces activités associatives sur le développement local au Maroc, et aussi d'inscrire son évolution dans le cadre plus large de l'histoire de l'associationnisme des immigrés 
Répartition des associations marocaines en France par type (1996-2011)
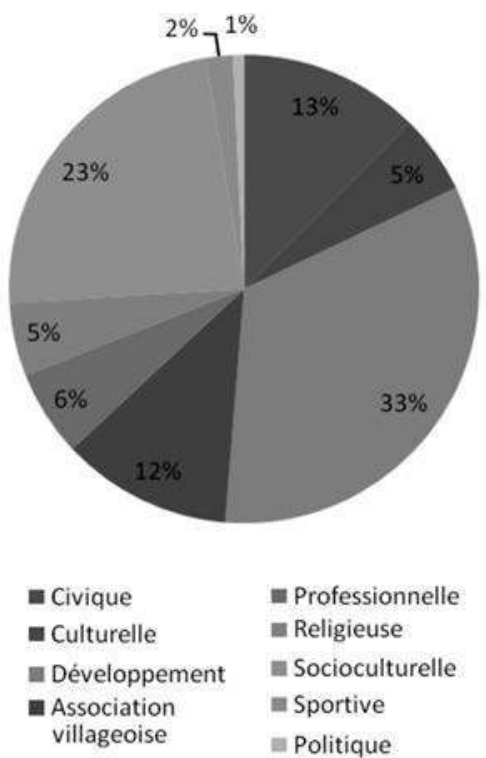

- Professionnelle

- Religieuse

an Socioculturelle

an Sportive

= Politique

que quatre catégories rassemblent plus de 80 \% des associations en France : les organisations civiques et socioculturelles, d'un côté, les organisations de développement et les associations villageoises, de l'autre. Les deux premières catégories travaillent en direction de la population marocaine établie en France. Elles constituent à la fois un espace d'intimité communautaire, d'entraide locale et de revendication des droits. Leur existence est liée aux besoins suscités par l'installation en France. À l'inverse, les organisations de développement et les associations villageoises ont avant tout une orientation transnationale. Elles répondent à un besoin de développement ressenti dans le pays d'origine. En regardant de plus près la structuration de ce champ et son évolution, il apparaît que le militantisme transnational n'émerge qu'au milieu des années 1990. C'est à partir de 2002-2003 que les organisations de développement ont supplanté, en termes de nombre de créations, les associations focalisées sur le pays d'accueil (voir graphique ci-après). Envi-

Source : Thomas Lacroix, $2012^{3}$

marocains en France. Pour ce faire, cette recherche s'appuie sur une analyse quantitative des associations de migrants marocains en France et de leurs activités. Elle apporte ainsi un nouvel éclairage sur un phénomène jusqu'ici documenté par des études qualitatives. Cette étude s'appuie sur une base de données des associations marocaines créées entre 1996 et 2011. Cette base est le produit d'un dépouillement systématique du Journal officiel, disponible en ligne. Elle inclut un descriptif de chaque association, de ses activités et de ses objectifs, sa date et son lieu de création. Les données fournies par le Journal officiel ont été complétées par une recherche Internet, ainsi que par une enquête approfondie auprès de 35 leaders associatifs. La base inclut 1599 organisations. Elle constitue, à ce jour, la base la plus complète. Le graphique ci-contre indique ron 70 organisations transnationales sont créées chaque année en France.

La structuration de ce champ associatif marocain en France porte non seulement la marque de l'histoire de l'installation de cette population, mais aussi l'histoire des relations entre le gouvernement marocain et les Marocains expatriés.

\section{Émergence et structuration du champ associatif marocain en France}

Le paysage des associations "localistes" est le fruit d'une longue histoire qui a ses racines dans la construction de l'État-nation marocain après l'indépendance. On distingue, d'abord, les organisations issues de la gauche marocaine en exil : l'Association des Marocains en France (AMF), 
créée en 1962 par Mehdi Ben Barka, et l'Association des travailleurs marocains en France (ATMF), créée en 1982 suite à une scission de l'AMF. À l'opposé du spectre politique se tiennent les Amicales des travailleurs et commerçants marocains en France, dont la première fut fondée en 1973 en région parisienne. Leur fonction principale était de servir de relais pour les consulats auprès de la population installée en France et d'animer la vie culturelle dans un souci de préservation du lien d'allégeance avec le pays d'origine. Toutefois, au-delà de ces activités socioculturelles, les Amicales ont fonctionné comme des agents de contrôle politique de la population en prévenant les implications syndicales et militantes de leurs membres ${ }^{4}$. La polarisation du champ associatif marocain et l'opposition souvent violente entre les deux camps en France ont durablement marqué les pratiques. Aujourd'hui, ces organisations comptent 68 représentations en France (49 Amicales, 16 pour l'ATMF et 3 pour l'AMF).

À côté de ces organisations historiques est apparu à partir des années 1990 tout un champ associatif affichant sa neutralité en refusant de s'inscrire dans la rivalité entre les associations gauchistes et les Amicales. Cette émergence d'un tiers secteur s'explique par la libéralisation de la politique de l'État marocain à l'égard de ses expatriés. Les partis de gauche au Maroc réintègrent le jeu politique parlementaire, tandis que l'opposition frontale entre sympathisants et opposants au régime prend fin. La participation associative des nationaux à l'étranger cesse d'être une question politique sensible.

Leurs activités couvrent un champ très large, depuis le montage d'expositions sur l'artisanat marocain, la célébration des fêtes religieuses ou civiques, la défense des droits, la lutte contre les discriminations jusquau soutien des membres pendant les périodes de difficulté (chômage, décès...). Sous l'étiquette "civique et sociocultu- relle", on trouve un éventail d'activités qui s'étend du culturel au politique, en passant par le religieux et le social. 199 associations civiques et 373 socioculturelles ont été créées depuis 1996. Elles constituent les secondeset troisième catégories les plus importantes après les associations de développement. Il faut noter le faible nombre d'organisations qui se déclarent politiques ou religieuses. Cela s'ex-

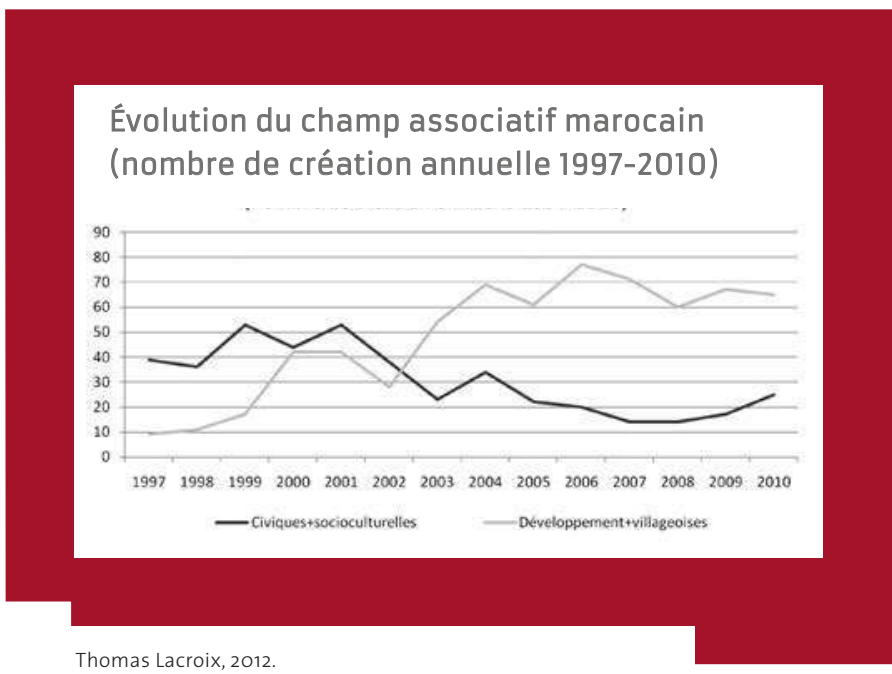

plique en grande partie par le contexte républicain français qui restreint les possibilités d'expression communautaire dans la sphère publique. Par ailleurs, sur le terrain, la vaste majorité des associations religieuses ne se déclarent ni ne se perçoivent comme spécifiquement "marocaines" dans la mesure où elles incluent des membres de diverses origines.

\section{Le tournant du développement}

Quant aux associations transnationales, elles sont la marque d'une mutation profonde du militantisme expatrié qui a débuté au tournant des années 2000. En soit, elles ne constituent pas une nou- 
Les amis et supporters de Najlae Lhimer, une lycéenne marocaine récemment expulsée de France comme immigrée illégale, manifestent le 13 mars 2010 à l'aéroport de Orly où ils attendent son retour de Casablanca. Najlae qui avait été arrêtée en février après avoir déposé plainte contre son frère, puis expulsée vers le Maroc. Elle a été autorisée à revenir en France par le président Nicolas Sarkozy @ AFP Pното Thomas Coex

veauté dans le champ associatif marocain. Jusque dans les années 1980, il existait un transnationalisme associatif que l'on peut qualifier de politique. Ainsi, la principale organisation, l'Association des Marocains de France, fut créée afin de soutenir les partis de gauche au Maroc. De même, les organisations villageoises ont toujours gardé une orientation transnationale d'aide et de soutien au village d'origine. Toutefois, ces dernières opéraient de manière informelle et leur fonction première a été avant tout l'accueil et l'installation des ruraux expatriés. Le transnationalisme développementaliste contemporain marque une profonde rupture

avec le militantisme passé. Son émergence soudaine a bouleversé le champ associatif marocain en l'espace d'une décennie. Au total, 950 associations déclarent participer à un projet de développement, soit $60 \%$ des associations marocaines en France. Et si l'on inclut toute forme d'activités transfrontalières en plus de celles de développement (activités économiques, socioprofessionnelles, religieuses, etc.), ce chiffre atteint 1005 organisations. Ce revirement n'est pas étranger à la réforme de la politique marocaine à l'égard des expatriés. Dans un souci de maximiser les retombées économiques de la migration sur le pays d'origine, le gouvernement met en place, à partir du milieu des années 1990, une série d'institutions et de politiques de cofinancement destinées à encourager les investissements et activités philanthropiques. La ques- 
tion du développement est un argument central au cour de la refondation du pacte social entre le Maroc et ses expatriés au sortir des "années de plomb”, qui sétendent des années 1970 aux années 1980. Le dernier avatar de cette évolution est la création du Conseil de la communauté des Marocains de l'étranger en 2007, instance consultative chargée de représenter les intérêts des Marocains dans le monde. Cette instance souffre cependant des anciennes dissensions militantes qui perdurent depuis les années 1980, signe que le développement ne peut, à lui seul, jouer le rôle de thématique fédératrice pour une "communauté" somme toute assez divisée. Parmi ces organisations versées dans le développement, on peut distinguer deux catégories. La première et la plus visible de ces trois catégories est celle des ONG de migrants. Les organisations de développement sont des organisations spécifiquement créées dans le but de conduire un projet de développement au Maroc. On dénombre 534 créations d'ONG de migrants depuis 1996. Soit une création sur trois au cours de la période. Elles sont de natures très diverses. Une majorité est constituée de petites associations finançant l'envoi de vêtements ou de matériel médical, soutenant un orphelinat ou une école. Leur durée de vie peut être très courte, ne dépassant pas celle du projet luimême. Leur bureau est souvent mixte, incluant des personnes sans ascendances marocaines. Ce sont des amis, des voisins sensibilisés aux problèmes du Maroc par les migrants. Toutefois, au-delà de ce tissu local relativement fragile, il existe un nombre important d'organisations pérennes qui ont su bénéficier du soutien des pouvoirs publics.

La seconde catégorie d'organisations transnationales est celle des associations villageoises. Cellesci ont fortement évolué au cours du temps. Après avoir été un soutien pour les nouveaux arrivants, elles sont devenues, avec l'épuisement des filières migratoires, des espaces de sociabilité où se partagent les informations sur la vie des membres (mariages, naissances, décès) et du village, et où sont gérées des caisses de solidarité pour les membres ou pour les besoins du village d'origine. Ces caisses ont permis aux expatriés de continuer à pourvoir aux charges collectives coutumières (tiwizi) du village telles que la restauration des systèmes d'irrigation ou des bâtiments religieux. À partir des années 1990, ce savoir-faire en matière de solidarité transnationale a été mobilisé pour la conduite de projets de développement. Aujourd'hui, on observe un grand nombre d'associations villageoises se dotant de statuts officiels afin de pouvoir mener à bien des projets tels que l'électrification, la construction d'une école ou la mise en place de cours d'alphabétisation (188 créations sur la période).

Enfin, il faut souligner que le transnationalisme déve-
Les organisations de développement sont des organisations spécifiquement créées dans le but de conduire un projet de développement au Maroc. On dénombre 534 créations d'ONG de migrants depuis 1996.

\section{loppementaliste concerne} de plus en plus des associations locales. À côté de leurs activités à destination de la population en France, un grand nombre accordent une partie de leur temps et de leurs ressources à un projet pour le Maroc. Certaines mènent ces activités de façon parallèle. La plupart parviennent à combiner les deux niveaux en étendant à léchelle transnationale leur travail au niveau local : associations faisant la promotion d'artistes marocains, associations d'informaticiens proposant des formations dans des écoles, associations de défense des droits soutenant une campagne de revendication à l'étranger, association de promotion de l'intégration organisant des chantiers internationaux, etc. C'est, par exemple, le cas de Asshab (Tours), créée en 2008 dans le but de faciliter l'insertion des jeunes dans la vie économique et politique locale. Asshab a participé à la mise en place d'un jumelage entre les universités de Tours et de Rabat, ainsi qu'à un projet de commercialisation d'herbes aromatiques. Chacune de ces initiatives fut conduite en associant des jeunes de l'agglomération dans une optique d'insertion. 


\section{Les principales associations de développement}

Migrations et développement est la plus ancienne et la plus importante des associations de ce type. L'association est inséparable de son fondateur, Lahoussain Jamal. Il émigre à l'âge de 17 ans en France où il trouve un emploi à l'usine de Trévisol (groupe Péchiney), dans les Hautes-Alpes, à l'Argentière-la-Bessée. Il adhère à la CFDT en 1972 et devient membre du conseil d'administration de l'usine. L'unité entame sa fermeture progressive en 1984 et offre aux ouvriers marocains, algériens et tunisiens une prime de retour de 250000 francs. Dans ce contexte, Jamal Lahoussain fonde l'association Retour et développement en 1986, qui devient Migrations et développement en 1987. Au cours des années 1990, l'association met en place sa méthode de travail. Elle intervient à la demande d'un collectif villageois structuré en association incluant ses émigrés en France. L'association fournit dès lors un appui tech-
Immigration, développement, démocratie (IDD) fut fondée

à l'issue d'un débat de plusieurs années au sein de la gauche marocaine en Europe, en avril 1999. Il s'agit moins d'une organisation centrale que d'un réseau au sein duquel siègent diverses catégories d'acteurs: associations, techniciens, experts... nique et financier au groupe ainsi constitué. Au fil des ans, l'association s'est positionnée en principal intermédiaire entre les bailleurs de fonds publics et privés, d'une part, et les porteurs de projets du Sud marocain, de l'autre. À partir de 1993, Migrations et développement va décrocher une série de partenariats. Son budget, qui était de 193000 francs en 1991, passe à 1,7 million de francs en 1994 et à 3,9 millions de francs en 1995. Ainsi, l'association élargit le champ de ses activités sur l'ensemble de la région de Taliouine, dont ses membres sont originaires. La manne budgétaire permet à Migrations et développement d'établir un plan pluriannuel d'électrifi- cation. L'association s'inscrit dans un programme spécial de raccordement pour la province de Taroudannt, dont l'objectif est d'électrifier $80 \%$ de la province en cinq ans. À partir de 1998, le ministère marocain de l'Éducation nationale associe Migrations et développement à un programme d'éducation informelle. L'association prend en charge la construction et l'équipement de 13 locaux, répartis sur 12 communes et 2 provinces. Léducation informelle entend pallier le manque d'infrastructures publiques. Outre l'alphabétisation, elle inclut un volet de préformation professionnelle proposant, par exemple, une initiation à la broderie, etc. L'association investit, par ailleurs, le domaine de léquipement hydraulique. Le programme de mobilisation des eaux de surface, étalé d'octobre 1996 à octobre 2000, a permis la réalisation, répartie sur 27 villages, de 10 retenues collinaires ${ }^{5}, 12$ bassins d'accumulation, $1,5 \mathrm{~km}$ de seguias, $12 \mathrm{~km}$ de canalisations, 6 puits, 8 pompes et tous les ouvrages d'aménagement (fontaines, lavoirs, etc.).

Immigration, développement, démocratie (IDD) fut fondée à l'issue d'un débat de plusieurs années au sein de la gauche marocaine en Europe, en avril 1999. Il s'agit moins d'une organisation centrale que d'un réseau au sein duquel siègent diverses catégories d'acteurs : associations, techniciens, experts... Les membres sont donc soit des associations de migrants dont l'un des membres fait le lien avec l'ATMF (Attacharouk, Annakhil, Wartas Solidarité et Développement, et Oued Maït en région parisienne), soit les sections de l'ATMF qui consacrent une partie de leurs activités à un projet de développement (Mosaïque à Paris, les sections de Saint-Avold, Vauréal, Nancy, Argenteuil et Aix-en-Provence). À ces structures s'ajoutent trois organisations issues de la mouvance de Migrations et développement : Migration et codéveloppement Alsace (MCDA) qui est l'ancienne antenne mulhousienne de Migrations et développement ; CAD Souss et Collectif Ilhmaten France Europe de développement (Cifed). Enfin, IDD Alsace (IDDA) est une organisation basée à Strasbourg composée 
de membres de MCDA, de l'ATMF et d'autres structures strasbourgeoises. Deux autres associations de la région parisienne, la Fédération des associations de Figuig et Khamsa, ont rejoint le réseau IDD en 2002, en même temps que les associations CAD Souss, Cifed et Wartas. Au cours de ces dernières années, le réseau s'est ouvert à de nouveaux membres : Amitiés abraysiennes sans frontières (AASF) (Orléans), Crépuscule (Angers). Les 2 rives franco-marocaines (Tourcoing), ESAF 54 (Marxeville), FES Enfance Solidarité (Dieppe), les Amis de Taourirt (Paris), Taddart (Paris). Au total, une vingtaine d'associations ont adhéré au réseau. La dimension "mise en réseau" domine l'action d'IDD aussi bien en France qu'au Maroc. Chaque organisme membre travaille au Maroc avec une association partenaire. Ces partenaires locaux sont disséminés sur l'ensemble du Maroc : au Nord (provinces de Chefchaouen, Oujda, Tanger), au Centre (régions de Rabat, Fès, Azrou, Khemisset), à l'Est (Beni Mellal,), au Sud (Tiznit, Ouarzazate, Tata, Chtouka Aït Baha, Goulimime). IDD a impulsé des relations horizontales entre les associations marocaines, notamment par le biais de rencontres régio- nales. Par exemple, la rencontre de Taqjijt a permis à huit associations partenaires du Souss-MassaDraa de se rencontrer en 2001. La mise en réseau se traduit enfin dans sa dimension Nord-Sud par plusieurs rencontres et séminaires. La première s'est tenue à Mehdia en août 2000 et a débouché sur l'élaboration et la signature d'une charte commune (la charte du développement culturel), qui expose les grandes lignes directrices de l'action des partenaires.

\section{L'impact des activités associatives sur le développement du Maroc}

Les activités au Maroc des associations basées en France sont de nature très diverses et ne concernent pas uniquement le développement à proprement dit. La majeure partie des actions sont des projets d'infrastructures (construction de routes, centres de santé, électrification, écoles, aménagement urbain, etc.). Le succès de ce type d'initiatives s'explique en partie par le soutien gou-

\section{Les pratiques transnationales des associations établies en France}

\begin{tabular}{|c|c|c|c|}
\hline & $\begin{array}{l}\text { Associations } \\
\text { villageoises }\end{array}$ & $\begin{array}{c}\text { ONG } \\
\text { de migrants }\end{array}$ & Autres \\
\hline Civique/droits/politique & $0 \%$ & $0 \%$ & $11 \%$ \\
\hline Culture/religion/sport & $2 \%$ & $1 \%$ & $24 \%$ \\
\hline Économique & $1 \%$ & $4 \%$ & $19 \%$ \\
\hline \multirow{5}{*}{$\begin{array}{c}\text { Développement social } \\
\text { y compris: } \\
\text { Éducation/enfance } \\
\text { Humanitaire } \\
\text { Santé }\end{array}$} & $97 \%$ & $96 \%$ & $46 \%$ \\
\hline & $10 \%$ & $33 \%$ & $0 \%$ \\
\hline & $4 \%$ & $16 \%$ & $18 \%$ \\
\hline & $3 \%$ & $15 \%$ & $2 \%$ \\
\hline & $N=188$ & $N=534$ & $N=206$ \\
\hline
\end{tabular}


vernemental. Une série de programmes publics cofinancent les projets d'électrification (Programme d'électrification rural généralisé) ou d'accès à l'eau potable (Programme d'accès généralisé à l'eau en milieu rural). Ces projets ont un impact très positif sur le développement du pays. Dans la province de Taroudannt, l'électrification en milieu rural est aujourd'hui pratiquement achevée, alors que seuls $18 \%$ des ménages en bénéficiaient en 1996. Ce progrès n'aurait pas été possible sans l'apport des expatriés et de leurs associations.

Les projets de développement humain (éducation, santé) sont, après les projets d'infrastructures, la deuxième forme d'intervention la plus fréquente. Ils incluent l'envoi de matériel scolaire et informatique, des bourses d'études aux familles nécessiteuses, l'organisation de cours d'alphabétisation pour les femmes (ou "éducation informelle"). Ces initiatives, malgré les besoins, ont un impact plus diffus sur la population au Maroc. Leurs effets ne se feront sentir quà plus long terme. De plus, contrairement aux projets d'infrastructures, ils ne sont adossés à aucun plan

Ces projets ont un impact très positif sur le développement

du pays. Dans la province de Taroudannt, l'électrification en milieu rural est aujourd'hui pratiquement achevée, alors que seuls $18 \%$ des ménages en bénéficiaient en 1996.

humanitaires (vêtements, jouets...), en particulier lors de catastrophes naturelles. Ce type d'opérations est avant tout conduit par de petites organisations philanthropiques dont les membres sont, certes, pleins de bonne volonté mais manquent de compétences professionnelles. Leur impact est de court terme.

Il faut noter un partage des tâches entre les associations transnationales qui consacrent plus de $95 \%$ de leurs activités au développement social (infrastructures, santé, éducation, etc.), d’une part, et les associations "localistes"s dont les pratiques au Maroc sont beaucoup plus diversifiées. Ces dernières sont, en particulier, plus impliquées dans les projets de nature économique (19 \% de leurs activités transnationales) : aide à l'investissement, constitution de réseaux socioprofessionnels (Association marocaine d'économie financière à Paris, Club des investisseurs marocains de l'étranger à Mérignac). Ces projets économiques sont le fait soit d'organisations professionnelles, soit d'associations d'aide à l'insertion. Les premières (associations d'étudiants ou de travailleurs diplômés tels que des médecins, des informaticiens, etc.) sont utilisées par leurs membres soit dans un but d'échange et de formation, soit pour élargir leurs débouchés (aide à la création d'entreprises, partenariats économiques, etc.). Les secondes utilisent les liens transnationaux à des fins d'insertion professionnelle (organisation de stages, chantiers, etc.). L’exemple de Asshab à Tours a déjà été mentionné. On peut également citer l'Association humanitaire et solidaire union franco-marocaine à Sottevillelès-Rouen, l'Association montpelliéraine citoyenne et culturelle à Montpellier et l'Association culturelle et sportive franco-marocaine à Belfort.

À l'inverse, on observe un faible engagement des opérateurs transnationaux (ONG et associations villageoises) dans le domaine économique (respectivement $1 \%$ et $4 \%$ de leurs activités). Cela peut s'expliquer par trois facteurs. En premier lieu, ces acteurs ne disposent pas du savoir-faire particulier (ni de la motivation) requis pour ce type de projets. Les acteurs associatifs ne sont pas nécessairement des entrepreneurs. En second lieu, les projets à caractère économique les conduiraient nécessairement à empiéter sur le champ des intérêts privés, ce qui, et c'est en particulier vrai pour les associations villageoises, affecterait la crédibilité et le consensus dont elles ont besoin auprès des acteurs locaux pour travailler. En dernier lieu, ces acteurs travaillent en majorité dans des zones rurales dont les besoins en développement se déclinent prioritairement en termes humains (déficit de formation) et d'in- 
frastructures. Encourager l'investissement et la création d'entreprises dans des régions qui ne disposent ni de la main-d'œuvre adéquate, ni de routes ou d'électricité serait un non-sens. Cette division du travail entre opérateurs apparaît donc cohérente.

\section{Des organisations surtout actives en milieu rural}

L'impact spatial des pratiques associatives au Maroc se calque sur une géographie de l'émigration. En effet, les régions bénéficiant des projets associatifs sont essentiellement les zones d'origine des migrants vers la France. Nous avons pu recenser les zones d'activités de 319 associations. Près d'un projet sur trois (29\%) est réalisé dans le Souss-MassaDraa, foyer historique des migrations vers la France depuis la période coloniale. Les autres sont le Haouz (10 \%), l'Oriental (11\%), le Rif (8\%) et enfin le Tadla Azilal (6 \%). À l'exception de Marrakech et d'Essaouira, les projets conduits en zone urbaine sont relativement peu nombreux. Par exemple, seules 9 associations opèrent dans le Grand Casablanca. Il faut toutefois noter que ce recensement ne tient pas compte des associations qui déclarent travailler à l'échelle nationale, et non pour une région spécifique. C'est le cas de 23 d'entre elles. Ces organisations sont davantage susceptibles de travailler avec des partenaires publics et privés en zone urbaine. C'est le cas, par exemple, de l'association Solidarité franco-marocaine pour l'enfance (Sofmae), qui a établi des liens avec divers médecins et hôpitaux à Rabat, à Casablanca, à Salé ou à Sidi Kacem.

La division du travail relevée ci-dessus entre organisations transnationalistes et localistes est aussi une division géographique. Les organisations transnationalistes (de développement et villageoises) interviennent en majorité en milieu rurale. À l'inverse, les autres sont plus enclines à opérer en milieu

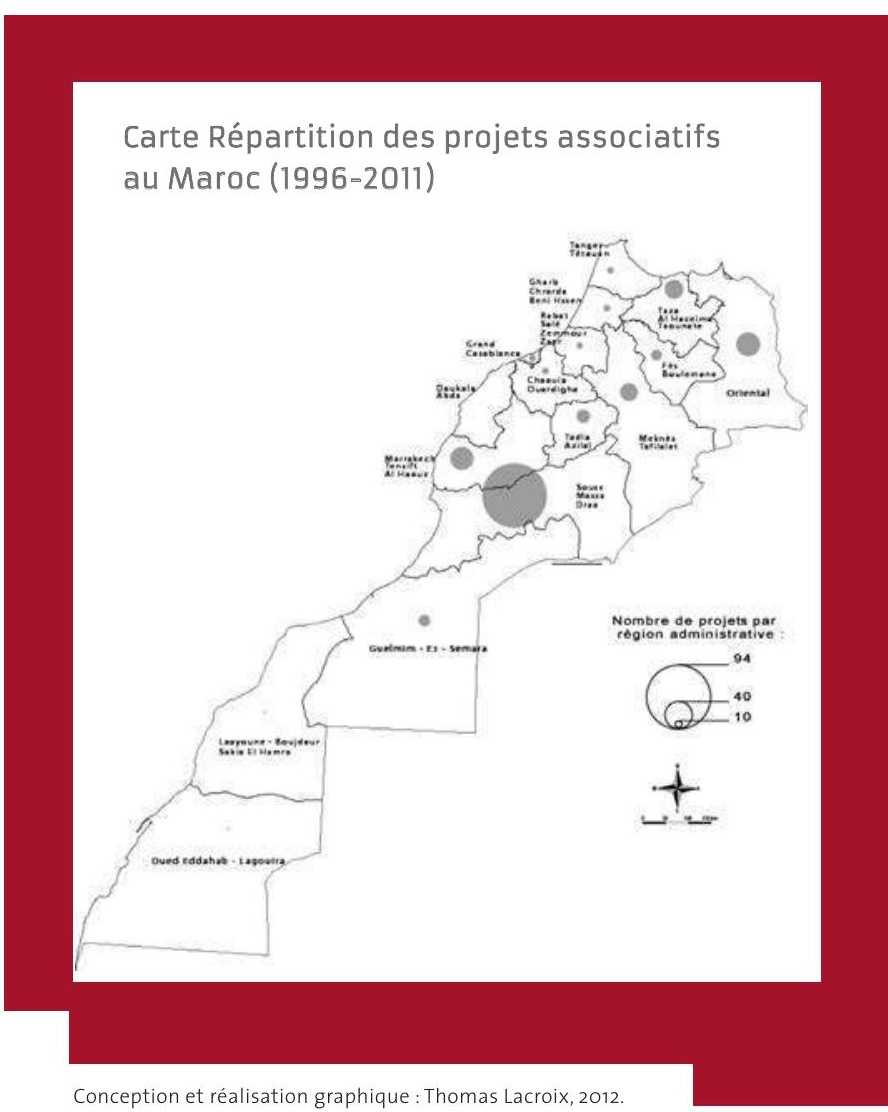

Source : Journal Officiel, enquêtes personnelles.

urbain. Ainsi, dans les provinces à forte population rurale telles qu'El Haouz, Azilal, Al Hoceima, Ouarzazate ou Beni Mellal, on relève pour chacune une seule association "localiste" déclarant y avoir des contacts. Dans le Grand Casablanca, 8 associations sur 9 sont des organisations sportives, culturelles ou socioprofessionnelles.

\section{Conclusion}

L'enquête conduite sur les associations des migrants marocains en France révèle l'ampleur prise par les engagements développementalistes. Ce transnationalisme associatif, en plein essor il y a une décennie, occupe aujourd'hui la majeure partie du champ des associations d'immigrés 
marocains en France. Cette évolution est remarquable par sa rapidité. Contrairement à une idée préconçue, ce renforcement des liens avec le pays d'origine ne signe pas l'échec de l'intégration des populations immigrées en France. Ce glissement du localisme au transnationalisme s'explique par la dynamique d'intégration à la fois sociale, politique et économique qui a rendu moins pressants les besoins liés à l'insertion dans la société d'accueil. De même, la nouvelle composition de l'immigration de travail, plus qualifiée que les migrations ouvrières des années 1970 et 1980, ne renouvelle pas la physionomie ouvrière et les besoins sociaux de la population marocaine en France. Si l'engage- ment transnational est la résultante de l'intégration dans le pays d'accueil, il peut aussi, en retour, la renforcer. En France, les liens qui se nouent entre ONG, pouvoirs publics et associations de migrants conduisent à une plus grande insertion de ces dernières dans la société civile. De plus, elles produisent une image positive de la migration, car adossée sur le développement, dans la sphère publique. L'intégration n'est donc pas incompatible avec un engagement transnational accru. Bien au contraire, les ressources sociales et financières accumulées grâce à l'insertion dans le pays d'accueil ont largement contribué à la sophistication des pratiques transnationales. 